\title{
Comunidade de aves no clube recreativo da SANEAGO em Iporá, Estado de Goiás
}

\author{
Aline Silva Teles ${ }^{1}$ \\ Daniel Blamires ${ }^{1 *}$ \\ Elivânia dos Santos Reis ${ }^{2}$ \\ ${ }^{1}$ Curso de Graduação em Biologia, Unidade Universitária de Iporá, Universidade Estadual de Goiás \\ Av. R-2, Q. 1, L. 1, Jardim Novo Horizonte II, CEP 76200-000, Iporá - Goiás, Brasil \\ ${ }^{2}$ Grupo de Pesquisa em Ecologia e Conservação de Aves (EcoAves) \\ Universidade Federal do Tocantins, Campus de Palmas - TO, Brasil \\ * Autor para correspondência \\ danbla1@yahoo.com.br
}

Submetido em 24/10/2011

Aceito para publicação em 19/04/2012

\section{Resumo}

Estudos com aves em ecossistemas urbanos brasileiros aumentaram nos últimos anos. Porém, abordagens desta natureza são incomuns no Cerrado. Assim, este trabalho inspecionou a estrutura da comunidade de aves no Clube Recreativo da SANEAGO, na malha urbana de Iporá, no centro-oeste goiano. As amostragens foram realizadas semanalmente entre abril de 2009 a março de 2010, totalizando 48 turnos. Registramos um total de 101 espécies, distribuídas em 35 famílias, sendo Tyrannidae a família com maior riqueza. Contudo, o estimador Jacknifel estimou uma riqueza de 120. A inspeção das categorias tróficas demonstrou um predomínio de espécies onívoras $(35,6 \%)$, insetívoras $(31,6 \%)$ e granívoras (10,9\%). A análise das categorias de estratificação vertical da vegetação demonstrou um predomínio de espécies arbóreas $(\mathrm{R}=81)$ e arbustivas $(\mathrm{A}=49)$, tal como esperado para a avifauna predominantemente arborícola do Cerrado. Este estudo demonstrou que o Clube possui uma avifauna rica, sendo assim importante para a manutenção e conservação da avifauna de Iporá.

Palavras-chave: Cerrado; Comunidade; Ecologia urbana; Estado de Goiás

\section{Abstract}

The bird community in the SANEAGO Recreation Club, in Iporá, Goiás. Studies of birds in Brazilian urban ecosystems have increased in recent years, however, works in the Cerrado are uncommon. This study surveyed the community structure of birds in the SANEAGO Recreation Club, in an urban area of Iporá, in mid-western Goiás. Samples were collected weekly between April 2009 and March 2010, totaling 48 sampling times. We found a total of 101 species in 35 families; Tyrannidae was richest. However, the estimator Jacknife 1 revealed 120 species for the study area. Inspection of the trophic categories showed a predominance of omnivores $(35.6 \%)$, insectivores $(31.6 \%)$ and granivores $(10.9 \%)$. An analysis of the categories of vertical stratification of vegetation showed a predominance of tree $(R=81)$ and shrub $(A=49)$ species, as expected for an area of Cerrado where there are predominantly arboreal birds. This study demonstrated that the bird diversity at the Club is rich, and that this area is important for the maintenance and conservation of the avifauna of Iporá.

Key words: Cerrado; Community; Goiás; Urban Ecology 


\section{Introdução}

A população humana aumentou de forma exponencial nas últimas décadas, concentrandose principalmente nos grandes centros urbanos (BEARDSLEY et al., 2009; FULLER et al., 2009). Dessa forma, os processo de urbanização modifica a paisagem natural e novos ambientes são criados de forma difusa, caracterizados por manchas de vegetação nativa, intercaladas com áreas de diferentes níveis de ocupação humana (BLAIR, 1996; MARZLUFF et al., 2001).No geral, esse processo afeta os padrões da biodiversidade e acelera as extinções em nível local e global (GASTON, 2005; MACGREGOR-FORS et al., 2009). Embora, cada cidade apresente um sistema único e complexo de ocupação, entender quais os efeitos da urbanização sobre a biodiversidade é fundamental no direcionamento de medidas efetivas para gestão e conservação das espécies (MARZLUFF et al., 2001; MACGREGOR-FORS et al., 2009).

Em ecossistemas urbanos as aves ocupam considerável destaque nas pesquisas, e seus estudos são importantes para subsidiar informações para a preservação dos hábitats, constatar o surgimento de espécies sinântropas e o desaparecimento de espécies incapazes de se adaptarem aos hábitats transformados (MATARAZZO-NEUBERGER, 1998). Ademais, as aves fazem parte da paisagem urbana, constituindo-se num elemento natural neste ambiente antropizado (ARGELDE-OLIVEIRA, 1996). Nos últimos anos os efeitos da urbanização sobre este grupo foram amplamente estudados, e diversos fatores foram considerados para explicar a sua diminuição nestas paisagens com elevado impacto humano (MARZLUFF, 2001; MARZLUFF et al., 2001; MACGREGOR-FORS et al., 2009).

Assim, nos últimos anos diversos estudos foram desenvolvidos em distintas áreas urbanizadas brasileiras, tais como jardins públicos, campi universitários, avenidas, praças, parques e lagos (MATARAZZONEUBERGER, 1995; MONTEIRO; BRANDÃO, 1995; ALVES; PEREIRA, 1998; BORGES; GUILHERME, 2000; GIMENES; ANJOS, 2000; MENDONÇA-LIMA; FONTANA, 2000; FRANCHIN; MARÇAL-JÚNIOR, 2004; SILVA; BLAMIRES, 2007). Além da composição específica, estes estudos abordaram padrões de riqueza, abundância, diversidade e frequência de ocorrência. Em geral, estes trabalhos também enfocam aspectos conservacionistas, relativos à ação antrópica sobre cada avifauna estudada. Contudo, poucos estudos ecológicos foram desenvolvidos com aves no Estado de Goiás, seja em áreas legais de proteção (TUBELIS, 2004; RAGUSA-NETO, 2008), áreas de menor antropização em relação às paisagens urbanas (BLAMIRES et al., 2001; MOURA et al., 2005; CURCINO et al., 2007), e áreas inseridas em ambiente urbano (MONTEIRO; BRANDÃO, 1995; SILVA; BLAMIRES, 2007; PEREIRA; SILVA, 2009).

Ademais, sabe-se ainda menos com relação a aspectos ecológicos da avifauna na microrregião do centro-oeste goiano, tanto nas paisagens naturais quanto nas urbanizadas. Recentemente, Silva e Blamires (2007) analisaram a avifauna do lago municipal em Iporá, onde inexistiam estudos similares até então. Assim, neste estudo pretendemos ampliar o conhecimento da avifauna urbana do centro oeste goiano, a partir da análise da estrutura de uma comunidade de aves em outra localidade na periferia da malha urbana de Iporá.

\section{Material e Métodos}

\section{Área de estudo}

Conduzimos este estudo no Clube Recreativo da Companhia de Saneamento de Goiás (SANEAGO). O clube está localizado na periferia urbana do município de Iporá (16 $25^{\circ} 23^{\prime}$ 'S , 5106 '30'W, 610m de altitude), na microrregião centro-oeste de Goiás (Figura 1). O município possui cerca de 31.270 habitantes, numa área de $1.026 \mathrm{~km}^{2}$ (IBGE, 2010). A paisagem primária do entorno da malha urbana era caracterizada pela dominância de formações savânicas, intercaladas por florestas mesofíticas e pastagens nativas (SILVA et al., 2006). Devido à atividade pecuária das últimas décadas, atualmente o perímetro urbano de Iporá é cercado, principalmente, por pastagem exótica de Brachiaria sp. O clima regional é marcado por duas estações bem definidas: seca (maio a setembro) e chuvosa (outubro a abril) (ASSAD, 2001). 
FIGURA 1: Localização geográfica do município de Iporá, no Estado de Goiás.

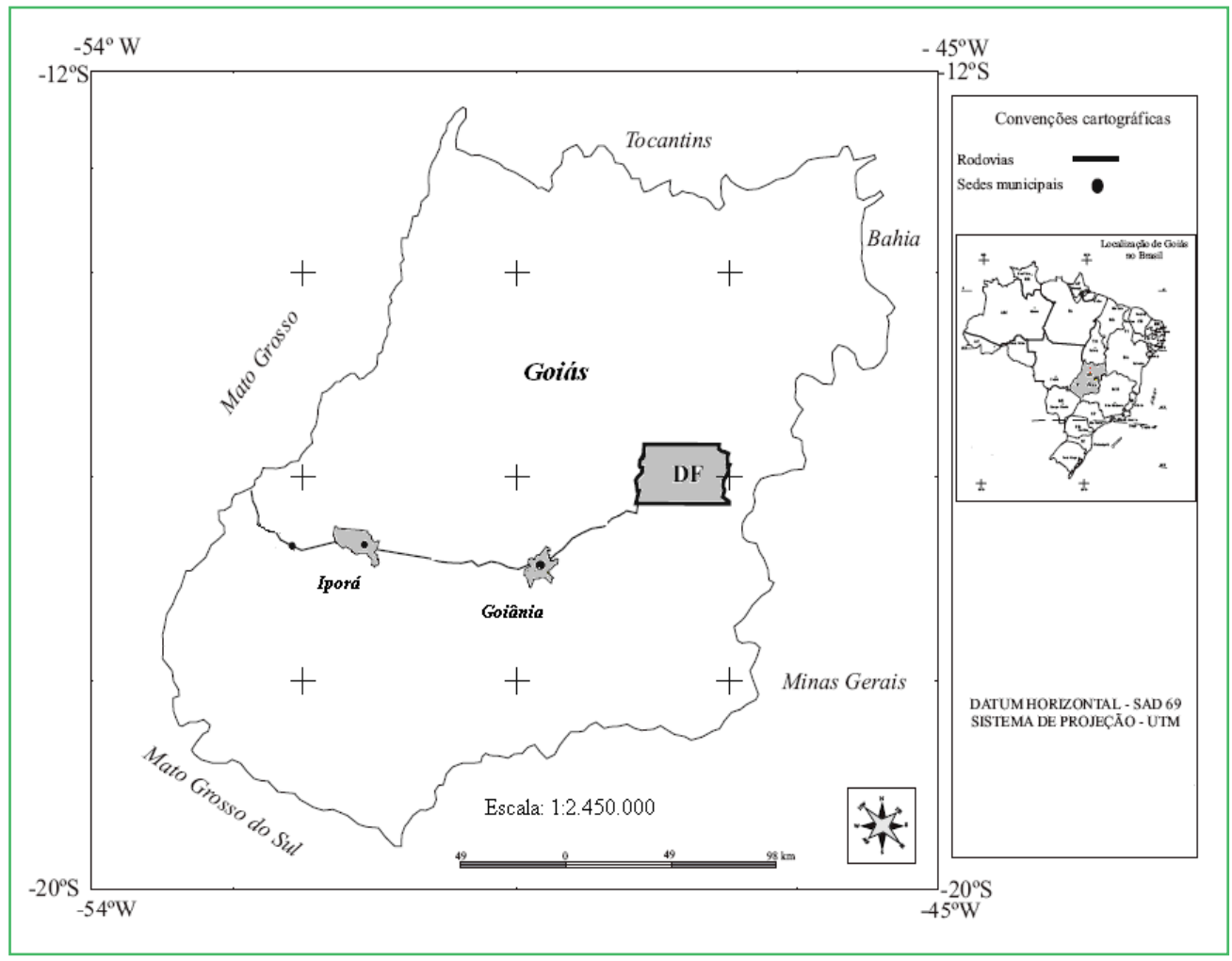

O Clube está inserido numa área com cerca de 4ha, tendo um perímetro de aproximadamente $700 \mathrm{~m}$. É constituído por um parque aquático, com duas piscinas, dois campos gramados de futebol e uma quadra de futebol de salão. O Clube possui uma pista de caminhada com aproximadamente $300 \mathrm{~m}$ de comprimento, posicionada em parte do perímetro. A vegetação do Clube é composta por espécies exóticas frutíferas e de sombreamento, como eucaliptais (Eucaliptus sp.) e sibipiruna Caesalpinia pluviosa DC., intercaladas sobre vegetações nativas herbáceo-arbustivas em crescimento secundário. Bananeiras (Musa sp.) e bambuzais (Bambuza sp.) também constituem a flora da área de estudo. O limite oeste do Clube é coberto por uma pequena extensão de Floresta Ciliar que margeia o córrego Tamanduá.

A vegetação no entorno do Clube é predominantemente composta por pastagens exóticas de capim braquiária (família Graminae), intercalados por manchas de Cerrado sentido lato ou árvores isoladas. A ocupação humana nos arredores do clube pode ser considerada baixa, sendo basicamente pequenos sítios para atividade pecuária leiteira e lavouras temporárias, as quais permanecem conectadas entre si e com o restante do município apenas por estradas vicinais.

\section{Metodologia}

Realizamos amostragens semanais entre abril de 2009 a março de 2010, totalizando 48 turnos. Cada turno teve início por volta das $06 \mathrm{~h} 30 \mathrm{~min}$, e era concluído aproximadamente às $07 \mathrm{~h} 20 \mathrm{~min}$. $\mathrm{O}$ procedimento de registro das espécies, baseado em Mendonça-Lima e Fontana (2000), consistiu em percorrer uma trilha no perímetro do Clube $(700 \mathrm{~m})$. Os registros feitos tanto visualmente, com o uso de binóculos 20x50mm e 10x50mm, quanto pela identificação das vocalizações. Usamos Ridgely e Tudor (1989; 1994), Sick (1997) e Sigrist (2006) para determinar a identidade das espécies. Os nomes científicos e a classificação taxonômica seguiram o Comitê Brasileiro de Registros Ornitológicos (CBRO, 2010).

Foi gerada uma curva da riqueza observada de espécies a partir do método Mao Tau de reamostragem 
(COLWELL et al., 2004). Para estimar o número de espécies existentes nas dependências do Clube, utilizouse o estimador não-paramétrico Jacknife1. Ambos os procedimentos, gerados com 1000 aleatorizações, foram elaborados com o programa EstimateS 8.2.0 de Colwell (2009). É importante ressaltar que, entre os oito estimadores mais recomendados pela literatura, o Jacknifel apresenta melhor acurácia dos dados (HORTAL et al., 2006).

As espécies foram agrupadas em categorias tróficas, segundo informações sobre hábitos alimentares obtidas em Willis (1979), Motta-Júnior (1990), Sick (1997), Junniper e Parr (1998) e Nascimento (2000). Foram consideradas as seguintes categorias: insetívoros (INS), onívoros (ONI), frugívoros (FRU), granívoros (GRA), nectarívoros (NEC), carnívoros (CAR) e detritívoros (DET).

Finalmente, verificamos a distribuição das espécies no gradiente de estratificação vertical da vegetação, com base nas seguintes categorias (adaptado de MATTARAZO-NEUBERGER, 1995; VALADÃO et al., 2006a):

a) Epigeo (E): sobre o solo. Registros em gramados, pavimentos, ou no solo nu;

b) Herbáceo $(\mathrm{H})$ : consistiu no registro de espécies posicionadas a até $0,5 \mathrm{~m}$ de altura;

c) Arbustivo (A): entre 0,51 a 2,0m de altura, o que envolveu, por exemplo, espécies posicionadas tanto na vegetação arbustiva quanto em árvores de médio porte;

d) Arbóreo (R): espécies pousadas no substrato superior, a mais de 2,0m de altura.

e) Aéreo (Ar): envolveu o registro de espécies em vôo baixo sobre a área de estudo.

\section{Resultados e Discussão}

Registramos 101 espécies, pertencentes a 35 famílias, no clube (Tabela 1).

TABELA 1: Espécies de aves registradas no Clube da SANEAGO em Iporá, entre abril de 2009 a março de 2010 . A sequência taxonômica e os nomes científicos seguem CBRO (2010). T: categorias tróficas (INS=insetívoros, ONI=onívoros, $\mathrm{FRU}=$ frugívoros, GRA=granívoros, $\mathrm{NEC}=$ nectarívoros, $\mathrm{CAR}=$ carnívoros e $\mathrm{DET}=$ detritívoros); $\mathbf{E V}$ : categorias de estratificação vertical da vegetação ( $\mathrm{E}=$ epigeo, $\mathrm{H}=$ herbáceo, $\mathrm{A}=$ arbustivo, $\mathrm{R}=$ arbóreo e $\mathrm{Ar}=$ aéreo).

\begin{tabular}{|c|c|c|}
\hline TAXA & $\mathbf{T}$ & EV \\
\hline \multicolumn{3}{|l|}{ TINAMIDAE } \\
\hline Crypturellus parvirostris (Wagler, 1827) & ONI & $\mathrm{E}$ \\
\hline Rhynchotus rufescens (Temminck, 1815) & ONI & $\mathrm{E}$ \\
\hline \multicolumn{3}{|l|}{ ANATIDAE } \\
\hline Dendrocygna viduata (Linnaeus, 1766) & ONI & $\mathrm{Ar}$ \\
\hline \multicolumn{3}{|l|}{ ARDEIDAE } \\
\hline Syrigma sibilatrix (Temminck, 1824) & ONI & $\mathrm{E}, \mathrm{R}$ \\
\hline Egretta thula (Molina, 1782) & ONI & $\mathrm{R}$ \\
\hline \multicolumn{3}{|l|}{ THRESKIORNITHIDAE } \\
\hline Mesembrinibis cayennensis (Gmelin, 1789) & ONI & $\mathrm{E}, \mathrm{R}, \mathrm{Ar}$ \\
\hline Theristicus caudatus (Boddaert, 1783) & ONI & $\mathrm{E}, \mathrm{Ar}$ \\
\hline \multicolumn{3}{|l|}{ CATHARTIDAE } \\
\hline Cathartes aura (Linnaeus, 1758) & DET & $\mathrm{Ar}$ \\
\hline Coragyps atratus (Bechstein, 1793) & DET & $\mathrm{Ar}$ \\
\hline \multicolumn{3}{|l|}{ ACCIPITRIDAE } \\
\hline Ictinia plumbea (Gmelin, 1788) & INS & $\mathrm{R}$ \\
\hline Rupornis magnirostris (Gmelin, 1788) & CAR & $\mathrm{R}$ \\
\hline \multicolumn{3}{|l|}{ FALCONIDAE } \\
\hline Caracara plancus (Miller, 1777) & CAR & $\mathrm{Ar}$ \\
\hline Falco sparverius Linnaeus, 1758 & CAR & $\mathrm{Ar}$ \\
\hline
\end{tabular}


Falco femoralis Temminck, 1822

CAR

$\operatorname{Ar}$

RALLIDAE

Aramides cajanea (Statius Muller, 1776)

ONI

E

CHARADRIIDAE

Vanellus chilensis (Molina, 1782)

ONI

E, Ar

COLUMBIDAE

Columbina talpacoti (Temminck, 1811)

GRA

E, H, A, R, Ar

Columbina squammata (Lesson, 1831)

GRA

E, H, A, R, Ar

Patagioenas picazuro (Temminck, 1813)

GRA

Patagioenas cayennensis (Bonnaterre, 1792)

GRA

E, H, A, R, Ar

Leptotila verreauxi Bonaparte, 1855

GRA

$\mathrm{R}$

PSITTACIDAE

Ara ararauna (Linnaeus, 1758)

FRU

Orthopsittaca manilata (Boddaert, 1783)

FRU

Diopsittaca nobilis (Linnaeus, 1758)

FRU

Aratinga leucophthalma (Statius Muller, 1776)

FRU

Aratinga aurea (Gmelin, 1788)

FRU

Forpus xanthopterygius (Spix, 1824)

FRU

Brotogeris chiriri (Vieillot, 1818)

FRU

Pionus maximiliani (Kuhl, 1820)

Amazona amazonica (Linnaeus, 1766)

FRU

CUCULIDAE

Piaya cayana (Linnaeus, 1766)

FRU

E, A, R

Crotophaga ani Linnaeus, 1758

INS

INS

INS

Ar

Ar

Guira guira (Gmelin, 1788)

TROCHILIDAE

Phaethornis pretrei (Lesson \& Delattre, 1839)

NEC

$\mathrm{R}$

Eupetomena macroura (Gmelin, 1788)

Aphantochroa cirrochloris (Vieillot, 1818)

NEC

NEC

$\mathrm{A}, \mathrm{R}, \mathrm{Ar}$

$\mathrm{R}$

Anthracothorax nigricollis (Vieillot, 1817)

NEC

Chlorostilbon lucidus (Shaw, 1812)

NEC

$\mathrm{A}, \mathrm{R}, \mathrm{Ar}$

A, R, Ar

Ar

Ar

Thalurania furcata (Gmelin, 1788)

NEC

$A, R$

Amazilia fimbriata (Gmelin, 1788)

NEC

E, H, A, R, Ar

ALCEDINIDAE

Chloroceryle amazona (Latham, 1790)

ONI

E, H, A, R, Ar

MOMOTIDAE

Momotus momota (Linnaeus, 1766)

GALBULIDAE

Galbula ruficauda Cuvier, 1816

BUCCONIDAE

Monasa nigrifrons (Spix, 1824)

PICIFORMES

RAMPHASTIDAE

Ramphastos toco Statius Muller, 1776

Pteroglossus castanotis Gould, 1834

PICIDAE

Melanerpes candidus (Otto, 1796)

Colaptes melanochloros (Gmelin, 1788)

ONI

$\mathrm{R}$

Colaptes campestris (Vieillot, 1818)

ONI

INS

$\mathrm{H}, \mathrm{A}, \mathrm{R}, \mathrm{Ar}$

$A, R$

$\mathrm{R}, \mathrm{Ar}$

A

A

$\mathrm{R}, \mathrm{Ar}$

ONI

Ar

Celeus flavescens (Gmelin, 1788)

ONI

ONI

A, $\mathrm{R}, \mathrm{Ar}$

$\mathrm{R}$

INS

Ar

INS

$\mathrm{R}$

INS

INS

E, A, R, Ar

INS

$R$

A, R 
THAMNOPHILIDAE

Taraba major (Vieillot, 1816)

INS

E, $H, R$

Thamnophilus doliatus (Linnaeus, 1764)

INS

E, H, A, R, Ar

FURNARIIDAE

Furnarius rufus (Gmelin, 1788)

ONI

Certhiaxis cinnamomeus (Gmelin, 1788)

INS

E, H, R

TYRANNIDAE

Elaenia flavogaster (Thunberg, 1822)

ONI

ONI

Elaenia chiriquensis Lawrence, 1865

ONI

Camptostoma obsoletum (Temminck, 1824)

ONI

$\mathrm{R}$

Phaeomyias murina (Spix, 1825)

Pyrocephalus rubinus (Boddaert, 1783)

INS

Machetornis rixosa (Vieillot, 1819)

INS

Myiozetetes cayanensis (Linnaeus, 1766)

INS

Pitangus sulphuratus (Linnaeus, 1766)

ONI

Megarynchus pitangua (Linnaeus, 1766)

ONI

Griseotyrannus aurantioatrocristatus (d'Orbigny \& Lafresnaye, 1837)

INS

Tyrannus melancholicus Vieillot, 1819

INS

Tyrannus savana Vieillot, 1808

INS

Myiarchus tyrannulus (Statius Muller, 1776)

INS

$\mathrm{H}, \mathrm{A}, \mathrm{R}$

VIREONIDAE

Cyclarhis gujanensis (Gmelin, 1789)

ONI

$\mathrm{H}, \mathrm{A}, \mathrm{R}, \mathrm{Ar}$

Vireo olivaceus (Linnaeus, 1766)

ONI

$\mathrm{H}, \mathrm{A}, \mathrm{R}, \mathrm{Ar}$

$$
\mathrm{R}
$$

HIRUNDINNIDAE

Pygochelidon cyanoleuca (Vieillot, 1817)

INS

Stelgidopteryx ruficollis (Vieillot, 1817)

INS

E, H, A, R

Progne tapera (Vieillot, 1817)

Progne chalybea (Gmelin, 1789)

INS

E, H, R, Ar

$A, R$

E, H, A, R

$A, R$

$R$

E, A, R, Ar

Ar

$\mathrm{R}$

TROGLODYTIDAE

Troglodytes musculus Naumann, 1823

Cantorchilus leucotis (Lafresnaye, 1845)

DONACOBIIDAE

Donacobius atricapilla (Linnaeus, 1766)

POLIOPTILIDAE

Polioptila dumicola (Vieillot, 1817)

INS

A, $R$

E, H, A, R

TURDIDAE

Turdus leucomelas Vieillot, 1818

Turdus amaurochalinus Cabanis, 1850

MIMIDAE

Mimus saturninus (Lichtenstein, 1823)

COEREBIDAE

Coereba flaveola (Linnaeus, 1758)

THRAUPIDAE

Nemosia pileata (Boddaert, 1783)

Tangara sayaca (Linnaeus, 1766)

INS

INS

INS

$\mathrm{Ar}$

, Ar

$\mathrm{R}, \mathrm{Ar}$

E, A, R, Ar

Tangara palmarum (Wied, 1823)

INS

E, H, A, R

$\mathrm{H}, \mathrm{A}, \mathrm{R}$

Tangara cayana (Linnaeus, 1766)

ONI

ONI

$\mathrm{R}$

Dacnis cayana (Linnaeus, 1766)

ONI

A, R

Conirostrum speciosum (Temminck, 1824)

INS

E, H, A, R, Ar

EMBERIZIDAE

Ammodramus humeralis (Bosc, 1792)

INS

$\mathrm{R}$

ONI

ONI

ONI

ONI

INS

E, H, A, R

GRA

A, H, A, R

$\mathrm{R}$

E, H, R, Ar

$\mathrm{H}, \mathrm{A}, \mathrm{R}, \mathrm{Ar}$

E, H, A, R

$\mathrm{R}$

A, R

E, $\mathrm{H}$ 
Sicalis flaveola (Linnaeus, 1766)

Volatinia jacarina (Linnaeus, 1766)

Sporophila nigricollis (Vieillot, 1823)

Sporophila caerulescens (Vieillot, 1823)

Sporophila angolensis (Linnaeus, 1766)

PARULIDAE

Basileuterus hypoleucus Bonaparte, 1830

ICTERIDAE

Cacicus cela (Linnaeus, 1758)

Icterus pyrrhopterus (Vieillot, 1819)

Gnorimopsar chopi (Vieillot, 1819)

Molothrus bonariensis (Gmelin, 1789)

FRINGILLIDAE

Euphonia chlorotica (Linnaeus, 1766)

PASSERIDAE

Passer domesticus (Linnaeus, 1758)

$\begin{array}{cc}\text { GRA } & \text { E, H, A, R, Ar } \\ \text { GRA } & \text { E, H, A, R, Ar } \\ \text { GRA } & \text { E, H, A, R } \\ \text { GRA } & \text { E, H, R } \\ \text { GRA } & \text { R }\end{array}$

INS

A, R

ONI

ONI

A, R, Ar

ONI

$\mathrm{A}, \mathrm{R}, \mathrm{Ar}$

ONI

E, A, R, Ar

$\mathrm{E}, \mathrm{R}, \mathrm{Ar}$

ONI

R, Ar

ONI

E, H, A, R, Ar
A família Tyrannidae apresentou o maior número de espécies (14 ou 13,7\% do total), seguida por Psittacidae ( 9 ou $8,8 \%$ ) e Trochilidae ( 8 ou 7,8\%). Salientamos o registro de quatro espécies que normalmente não se ajustam a ambientes muito antropizados: arara-canindé Ara ararauna (Linnaeus, 1758), maracanã-do-burití Orthopsittaca manilata (Boddaert, 1783), araçarícastanho Pteroglossus castanotis Gould, 1834, e curió Sporophila angolensis (Linnaeus, 1766) (RIDGELY; TUDOR, 1989; SICK, 1997; JUNNIPER; PARR, 1998; RAGUSA-NETO, 2006; SIGRIST, 2006; GOULART et al., 2011).
A riqueza local de aves estimada foi de 120 espécies. A curva do estimador não-paramétrico Jacknifel mostrou que a estimativa para a riqueza de espécies apresenta uma mudança na pendente após o sexto mês de amostragem (Figura 2). Contudo, a riqueza não estabilizou, ou seja, o número de espécies continua aumentando até o final das amostragens (pendente final 1,25). Em geral, a riqueza de aves estimada no Clube da SANEAGO foi superior à riqueza detectada por Silva e Blamires (2007) em um Lago na área urbana de Iporá (70 espécies). Provavelmente a maior riqueza registrada no Clube deve-se a heterogeneidade dos ambientes, menor

FIGURA 2: Curva de riqueza estimada por Jacknifel e observada (Mao Tau), para a riqueza total de espécies registradas ao longo dos meses de amostragem de dados no Clube Recreativo da SANEAGO, em Iporá, centro-oeste goiano, entre abril de 2009 e março de 2010.

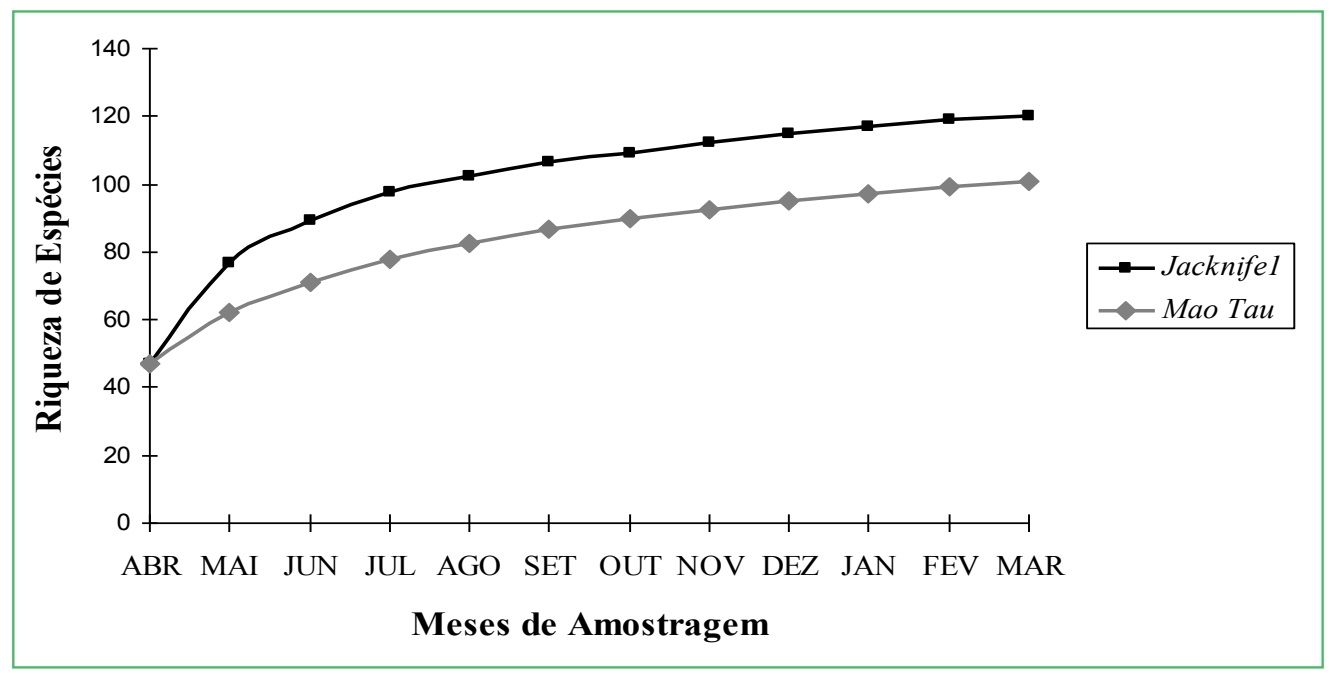


grau de ocupação humana do entorno e proximidade com a Mata Ciliar do Córrego Tamanduá.

Com relação às categorias tróficas, predominaram as espécies onívoras, insetívoras e granívoras (Tabela 2), o que também foi verificado noutras áreas urbanizadas brasileiras (ARGEL-DE-OLIVEIRA, 1995; MATARAZZO-NEUBERGER, 1995; SILVA; BLAMIRES, 2007; FUSCALDI; LOURES-RIBEIRO, 2008). Neste contexto, um aumento da onívoria é esperado em áreas antropizadas, as quais podem desempenhar um efeito tampão contra flutuações no estoque de alimentos (WILLIS, 1979; MOTTAJÚNIOR, 1990).

TABELA 2: Totais de espécies da avifauna do Clube Recreativo da SANEAGO (Iporá-GO), distintas segundo as categorias tróficas e respectivas proporções.

\begin{tabular}{cc}
\hline Categorias & Espécies (\%) \\
\hline Onívoros & $36(35,6)$ \\
Insetívoros & $32(31,6)$ \\
Granívoros & $11(10,9)$ \\
Frugívoros & $9(8,9)$ \\
Nectarívoros & $7(6,9)$ \\
Carnívoros & $4(3,9)$ \\
Detritívoros & $2(1,9)$ \\
\hline
\end{tabular}

A distribuição nos gradientes de estratificação vertical da vegetação demonstrou que a maioria das espécies concentrou-se nas categorias arbórea e arbustiva, respectivamente (Figura 3). Mais espécies pertencentes a estas categorias pode refletir uma característica das aves do Cerrado que, apesar de ser predominantemente coberto por savanas, possui uma avifauna com maioria das espécies de hábitos arborícolas (SICK, 1966). Contudo, esta maior preferência pode estar relacionada a distorções na metodologia, já que as aves tendem a buscar refúgio nos estratos mais altos quando percebem o observador (MATARAZZO-NEUBERGER, 1995; VALADÃO et al., 2006 a).
FIGURA 3: Distribuição das espécies do Clube Recreativo da SANEAGO (Iporá, Goiás, Brasil) nas categorias de estratificação vertical da vegetação. E: epigeu; $\mathrm{H}$ : herbáceo; A: arbustivo; R: arbóreo; Ar: aéreo.

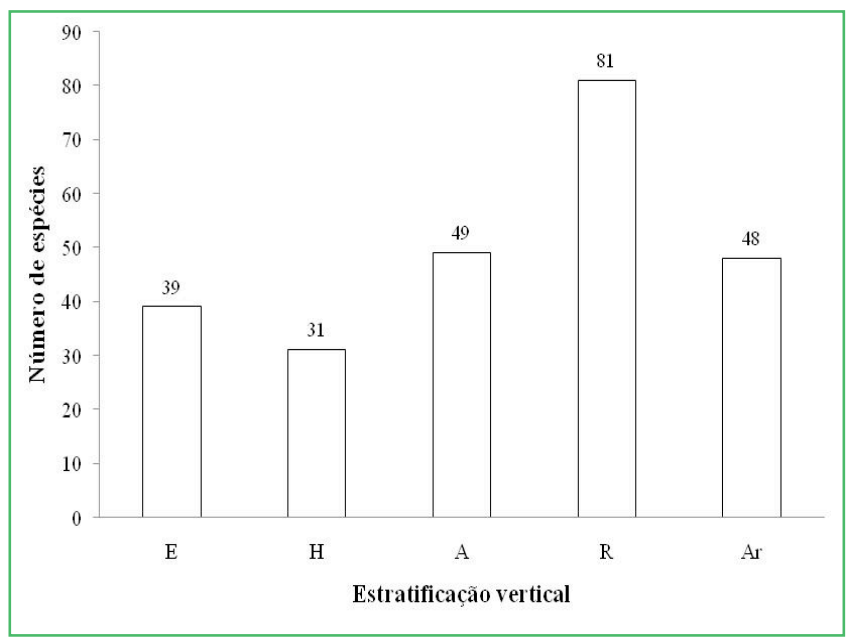

\section{Agradecimentos}

Agradecemos a Lázaro José da Cunha, Luzimar Magalhães de Rezende, e Aline Áthila Barros de Souza pelo apoio durante a atividade de campo. Célia Maria de Assis (Biblioteca UFG) contribuiu com a revisão bibliográfica. O Conselho Nacional de Desenvolvimento Científico e tecnológico (PIBIC/UEG/CNPq) concedeu uma bolsa de Iniciação Científica a Aline $\mathrm{S}$. Teles durante parte do desenvolvimento deste trabalho, e Flávio Alves de Souza confeccionou o mapa. Aos revisores anônimos que fizeram valiosas críticas nas versões anteriores do manuscrito.

\section{Referências}

ALVES, M. A. S; PEREIRA, E. F. Richness, abundance and seasonality of bird species in a lagoon of an urban área (Lagoa Rodrigo de Freitas) of Rio de Janeiro, Brazil. Ararajuba, Londrina, v. 6, n. 2, p. 110-116, 1998.

ARGEL-DE-OLIVEIRA, M. M. Aves e vegetação em um bairro residencial da cidade de São Paulo (São Paulo, Brasil). Revista Brasileira de Zoologia, Curitiba, v. 12, p. 81-92, 1995.

ARGEL-DE-OLIVEIRA, M. M. Aves urbanas. In: VIELLIARD, J. M. E.; SILVA, M. L.; SILVA, W. R. (Ed.). In: Anais do Congresso Brasileiro de Ornitologia,V, 1996, Campinas. Anais... Campinas: UNICAMP, 1996. p. 151-162.

ASSAD, E. D. Chuva no Cerrado: Análise e espacialização. 2. Ed. Brasília: EMBRAPA/SPI, 2001. CD-room.

BEARDSLEY, K.; THRONE, J. H.; ROTH, N. E.; GAO, S.; MCCOY, M. C. Assessing the influence of rapid urban growth and 
regional polices on biological resources. Landscape and Urban Planning, San Diego, n. 93, p. 172-183, 2009.

BLAIR, R. B. Land use and avian species diversity along an urban gradient. Ecological Applications, Ithaca, v. 6, p. 506-519, 1996.

BLAMIRES, D.; VALGAS, A. B.; BISPO, P. C. Estrutura da comunidade de aves da Fazenda Bonsucesso, município de Caldazinha, Goiás, Brasil. Tangara, Belo Horizonte, v. 1, n. 3, p. 101-113, 2001.

BORGES, S. H.; GUILHERME, E. Comunidade de aves em um fragmento florestal urbano em Manaus, Amazonas, Brasil. Ararajuba, Londrina, v. 8, n. 1, p. 17-23, 2000.

CBRO Comitê Brasileiro de Registros Ornitológicos. 2010. Disponível em <http://www.cbro.org.br/CBRO/index.htm>. Acesso em: 1 jul. 2010.

COLWELL, R. K. EstimateS: Statistical estimation of species richness and shared species from samples. Version 8. 2009. Disponível em <www.purl.oclc.org/estimates $>$. Acesso em: 10 jan. 2011.

COLWELL, R. K.; MAO, C. X.; CHANG, J. Interpolating, extrapolating, and comparing incidence-based species accumulation curves. Ecology, New York, n. 85, p. 2717-2727, 2004.

CURCINO, A.; SANT'ANA, C. E. R.; HEMING, N. M. Comparação de três comunidades de aves na região de Niquelândia, GO. Revista Brasileira de Ornitologia, São Leopoldo, v. 15, n. 4, p. 574-584, 2007.

FULLER, R. A.; TRATALOS, J.; GASTON, K. J. How many birds are there in a city of half a millon people? Diversity and Distributions, Stellenbosch, v. 15, p. 328-337, 2009.

FUSCALDI, R. G.; LOURES-RIBEIRO, A. A avifauna de uma área urbana do município de Ipatinga, Minas Gerais, Brasil. Biotemas, Florianópolis, v. 21, n. 3, p. 125-133, 2008.

FRANCHIN, A. G.; MARÇAL-JÚNIOR, O. A riqueza da avifauna no Parque municipal do Sabiá, Zona Urbana de Uberlândia (MG). Biotemas, Florianópolis, v. 17, n. 1, p. 179-202, 2004.

GASTON, K. J. Biodiversity and extinction: Species and people. Progress in Physical Geography, London, v. 29, p. 239-247, 2005. GOULART, F. F.; VANDERMEER, J.; PERFECTO, I.; DA MATTA-MACHADO, R. P. Frugivory by five bird species in agroforest home gardens of Pontal do Paranapanema, Brazil. Agroforestry Systems, Columbia, v. 82, n. 3, p. 239-246, 2011.

GIMENES, M. R.; ANJOS, L. Distribuição espacial de aves em um fragmento florestal do campus da Universidade Estadual de Londrina, Norte do Paraná, Brasil. Revista Brasileira de Zoologia, Curitiba, v. 17, p. 263-271, 2000.

HORTAL, J.; BORGES, P. A. V.; GASPAR, C. Evaluating the performance of species richness estimators: sensitivity to sample grain size. Journal of Animal Ecology, London, v. 75, p. 274-287, 2006.

IBGE- FUNDAÇÃO INSTITUTO BRASILEIRO DE GEOGRAFIA E ESTATÍSTICA. Cidades. 2010. Disponível em $<$ http://www.ibge.gov.br/cidadesat/default.php $>$. Acesso em: 10 jan. 2011.

JUNNIPER, T.; PARR, M. Parrots: A guide to the birds of the world. New Haven: Yale University Press, 1998. 584 p.

MACGREGOR-FORS, I.; ORTEGA-ÁLVAREZ, R.; SCHONDUBE, A. On the ecological quality of urban systems: An ornithological perspective. In: GRABER, D. S.; BIRMINGHAM,
K. A (Ed.). Urban Planning in the 21st Century. New York: Nova Science Publishers, 2009. p. 51-66.

MATARAZZO-NEUBERGER, W. M. M. Comunidades de aves de cinco parques e praças da grande São Paulo, Estado de São Paulo. Ararajuba, Londrina, v. 3, p. 13-19, 1995.

MATARAZZO-NEUBERGER, W. M. Ecossistema urbano: Considerações gerais e urbanização da avifauna no Brasil. In: Simpósio de Ecossistemas Brasileiros, IV, 1998, São Paulo. Anais... São Paulo: Academia de Ciências do Estado de São Paulo, 1998. p. 135-152.

MARZLUFF, J. M. Worldwide conservation and its effects on birds. In: MARZLUFF, J. M.; BOWMAN, R.; DONNELLY, R. (Ed.). Avian ecology and conservation in an urbanizing world. Massachussets: Kluwer Academic Publishers, 2001. p. 19-47.

MARZLUFF, J. M.; BOWMAN, R.; DONNELLY, R. 2001. Worldwide urbanization and its effects on birds. In: MARZLUFF, J. M., BOWMAN, R.; DONNELLY, R. (Ed.). Avian ecology and conservation in an urbanizing world. Massachussets: Kluwer Academic Publishers, 2001. p. 1-17.

MENDONÇA-LIMA, A.; FONTANA, C. S. Composição, frequência e aspectos biológicos da avifauna no Porto Alegre Country Clube, Rio Grande do Sul. Ararajuba, Londrina, v. 8, n. 1 , p. $1-8,2000$.

MONTEIRO, M. P.; BRANDÃO, D. Estrutura da comunidade de aves do "Campus Samambaia" da Universidade Federal de Goiás, Goiânia, Brasil. Ararajuba, Londrina, v. 3, p. 21-26, 1995.

MOTTA-JÚNIOR, J. C. Estrutura trófica e composição das avifaunas de três hábitats terrestres na região central do estado de São Paulo. Ararajuba, Londrina, v. 1, p. 65-71, 1990.

MOURA, N. G.; LARANJEIRAS, T. O.; CARVALHO, A. R.; SANTANA, C. E. R. Composição e diversidade da avifauna em duas áreas de Cerrado dentro do campus da Universidade Estadual de Goiás - Anápolis. Revista Saúde e Ambiente, Joinville, v. 6, n. 1, p. 34-39, 2005.

NASCIMENTO, J. L. X. Estudo comparativo da avifauna em duas Estações Ecológicas da caatinga: Aiuaba e Seridó. Melopsittacus, Belo Horizonte, n. 3, p. 12-35, 2000.

PEREIRA, K. D. L.; SILVA, R. Levantamento da avifauna da área urbana de Anápolis, Goiás. Ensaios e Ciência, Valinhos, v. 13, n. 2, p. 36-46, 2009.

RAGUSA-NETO, J. Dry fruits and the abundance of the blue-andyellow macaw (Ara ararauna) at a Cerrado remnant in Central Brazil. Ornitologia Neotropical, Montreal, v. 17, p. 491-500, 2006.

RAGUSA-NETO, J. Toco toucan feeding ecology and local abundance in an habitat mosaic in the brazilian Cerrado. Ornitologia Neotropical, Montreal, v. 19, p. 345-359, 2008.

RIDGELY, R. S.; TUDOR, G. The birds of South America, volume 1: The oscine passerines. Austin: University of Texas Press, 1989. $516 \mathrm{p}$.

RIDGELY, R. S.; TUDOR, G. The birds of South America, volume 2: The suboscine passerines. Austin: University of Texas Press, 1994. 814 p.

SICK, H. As aves do Cerrado como fauna arborícola. Anais da Academia Brasileira de Ciências, São Paulo, v. 38, n. 2, p. 355 $363,1966$. 
SICK, H. Ornitologia brasileira. Rio de Janeiro: Editora Nova Fronteira, 1997. 912 p. SIGRIST, T. Aves do Brasil: uma visão artística. São Paulo: Editora Avisbrasilis, 2006. 612 p.

SILVA, J. F.; FARIÑAS, M. R.; FELFILI, J. M.; KLINK, C. A. Spatial heterogeneity, land use and conservation in the Cerrado region of Brazil. Journal of Biogeography, New York, v. 33, p. 536-548, 2006.

SILVA, F. D. S.; BLAMIRES, D. Avifauna urbana no Lago Pôr do Sol, Iporá, Goiás, Brasil. Lundiana, Belo Horizonte, v. 8, n. 1, p. 17-26, 2007.

TUBELIS, D. P. Species composition and seasonal occurrence of mixed-species flocks of forest birds in central Cerrado, Brazil. Ararajuba, Londrina, v. 12, n. 2, p. 105-111, 2004.
VALADÃO, R. M.; FRANCHIN, A. G.; MARÇAL-JÚNIOR, O. A avifauna no parque municipal Vitório Siquierolli, zona urbana de Uberlândia (MG). Biotemas, Florianópolis, v. 19, n. 1, p. 81-91, 2006a.

VALADÃO, R. M.; MARÇAL-JÚNIOR, O.; FRANCHIN, A. G. A avifauna no parque municipal Santa Luzia, zona urbana de Minas Gerais. Bioscience Journal, Uberlândia, n. 22, n. 2, p. 97-108, 2006b.

WILLIS, E. O. The composition of avian communities in remanescent woodlots in Southern Brazil. Papéis Avulsos de Zoologia, São Paulo, v. 33, p. 1-25, 1979. 